\title{
Stellar Midlife Crises: Challenges and Advances in Simulating Convection and Differential Rotation in Sun-like Stars
}

\author{
Nicholas J. Nelson, Charles Payne and Cameron Michael Sorensen \\ Dept. of Physics, California State University, Chico \\ Chico, CA, USA 95929-0202 \\ email: njnelson@csuchico.edu
}

\begin{abstract}
Low mass, main sequence stars like our Sun exhibit a wide variety of rotational and magnetic states. Observational and theoretical advances have led to a renewed emphasis on understanding the rotational and magnetic evolution of sun-like stars has become a pressing problem in stellar physics. We use global 3D convection and convective dynamo simulations in rotating spherical shells and with realistic stellar stratification to explore the behavior of "middle-aged" stars. We show that for stars with slightly less rotational influence than our Sun a transition occurs from solar-like (fast equator, slow poles) to anti-solar (slow equator, fast poles) differential rotation. We investigate this transition using two different treatments for the upper boundary of our simulations and we hypothesize that this transition from solar-like to anti-solar differential rotation may be responsible for observations of anomalously rapid rotation for stars older than our Sun.
\end{abstract}

Keywords. hydrodynamics, MHD, Sun: interior, stars: activity, stars: rotation

\section{The Challenges of Stellar Rotation and Convection}

One of the most pressing and exciting challenges of modern astrophysics is the search for habitable exoplanets. With recent discoveries of potentially habitable Earth-like planets around nearby stars including Proxima Centauri (Anglada-Escudé et al. 2016) and TRAPPIST-1 (Gillon et al. 2016), there is considerable interest to understand if these planets could support life. With advances in exoplanet detection on the horizon there with doubtless be many more such systems discovered in the near future. The potential for extensive observational follow-up of these systems, however, is largely limited for the coming decade to refinements in orbital characteristics, stellar activity, and possibly atmospheric absorptions spectra for transiting planets. There is still likely many years between the present and the ability to directly image most potential Earth-analogues. This begs for a general theoretical understanding of habitability that can be applied to all planets around any star.

A comprehensive "theory of habitability" is an enormous theoretical challenge. To date, the question of habitability has largely focused simply on the mean surface temperature of the planet. Taking the recent discovery of Proxima Centauri b as an example, we see that in this case a more detailed and systematic examination by the community have raised serious questions on issues such as orbital dynamics (Kane et al. 2017; Méndez \& Rivera-Valentín 2017), planetary mass and composition (Bixel \& Apai 2017), space weather (Garrafo et al. 2016; Airapetian et al. 2017), formation scenarios Coleman et al. (2017), magnetospheric dynamics (Luger et al. 2016), climate (Boutle et al. 2017), and convective dynamo action (Yadav et al. 2016). This volume attempts to at least begin to bring a coherent focus on questions of habitability across a wide range of fields. Here 


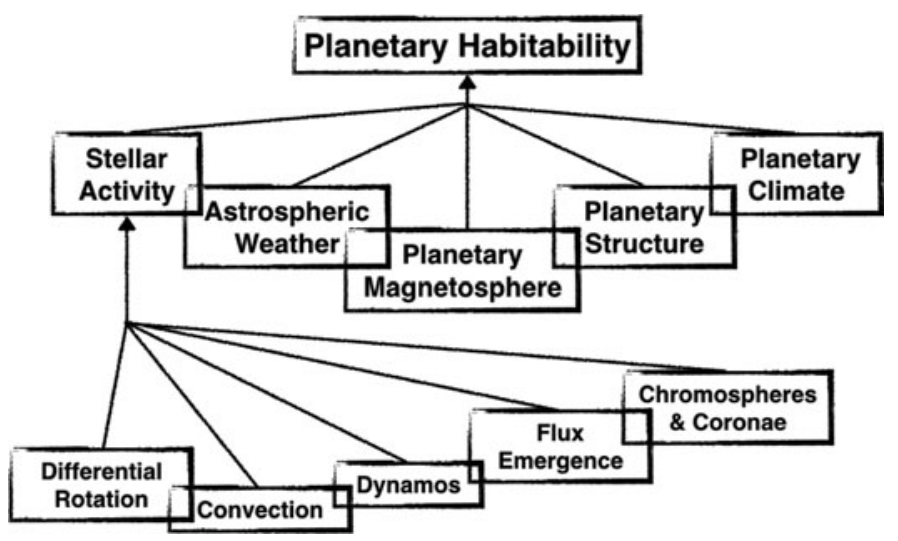

Figure 1. A schematic flow chart of how studies of stellar convection and differential rotation relate to questions of planetary habitability.

we will focus on the issue of stellar activity and even more specifically, on the modeling of convection and differential rotation in sun-like stars.

Figure 1 attempts to illustrate the broad range of inputs needed to theoretically predict the habitability of a particular planet around a particular star. While clearly incomplete, Fig. 1 highlights some of the topics from stellar interiors to climate that play key roles in habitability. Not shown but still of great importance are how these processes vary and evolve over geological and astrophysical timescales. In this paper focus on a specific contribution to the quest for a theory of habitability - the generation and emergence of stellar magnetism. We present simulations that explore recent advances in modeling convection and differential rotation in solar-like stars.

\subsection{Modeling Stellar Convection and Differential Rotation}

With the rapid increase in observational capabilities for both solar and stellar convection, rotation, differential rotation, and magnetic activity, there has been a corresponding advance in 3D numerical modeling which seeks to investigate the physical workings of these difficult-to-observe processes. As an example, after decades of searching, observers are possibly only now achieving detection of convective giant cells (e.g., Hanasoge et al. 2010, 2012; Hathaway et al. 2013; McIntosh et al. 2014; Greer et al. 2014, 2015). For other stars the observational guidance is even less clear and becomes progressively harder to interpret moving away from solar parameters such as age, mass, and rotation rate (see Reiners 2012).

Beginning with the work of Gilman (1983) and Glatzmaier (1985), 3D global convection and convective dynamo models have made key advances in improving understanding of the physical mechanisms which drive differential rotation, dynamo action, and magnetic activity in low-mass stars. Modeling stellar convection and dynamo action in 3D requires the use of advanced numerical techniques to utilize modern massively parallel computational resources. The simulations presented here use the ASH (Clune et al. 1999; Brun et al. 2004; Nelson et al. 2013b) and Rayleigh (Featherstone \& Hindman 2016a) codes. Both ASH and Rayleigh solve the equations of anelastic magnetohydrodynamics in rotating spherical shells with solar-like stratification. ASH simulations have been used to model solar-like differential rotation and meridional circulation (Brun \& Toomre 2002; Miesch et al. 2006; Featherstone \& Miesch 2015), convection and differential rotation in a variety of low-mass stars (Brown et al. 2008; Brun et al. 2017), and 
dynamo action in stars ranging from solar-like (Brown et al. 2010, 2011; Nelson et al. 2011, 2013b,a; Nelson \& Miesch 2014; Augustson et al. 2015) to core dynamos in highmass stars (Featherstone et al. 2009; Augustson et al. 2016) to some very low-mass stars (Browning 2008). Rayleigh has recently been developed to optimize the use of modern massively parallel computational architectures, enabling some of the highest-resolution simulations of stellar convection to date (Featherstone \& Hindman 2016b,a).

In this work we will not attempt to detail the variety of numerical methods used, but will refer the reader to published descriptions elsewhere in the literature in order to focus on presenting some recent results. Specifically, we will outline how the use of a stochastic plume boundary condition may permit an improved understanding of giant cell convection, how the degree of rotational influence yields sharp changes in differential rotation for solar-like stars, and how some of our least diffusive models achieve buoyant magnetic loops which rise through the convection zone and provide a window into the flux emergence process.

\section{Modeling Convection with a Plume Boundary Condition}

Even with continued exponential growth, computational resources provide strict bounds on the level of resolution a global-scale 3D convection simulation can achieve. This limit makes the inclusion of granular scales on the order of $1 \mathrm{Mm}$ beyond the reach of globalscale models for the foreseeable future. Even super-granular scales of $30 \mathrm{Mm}$ will require years of continued growth in computing resources. This means that for simulations with solar-like stratification the region above $0.98 R_{\odot}$ is inaccessible simply due to a lack of resolution. Thus modelers generally limit themselves to motions below that depth and place an impenetrable boundary near that point. One interesting alternative is to adjust the stratification to provide a stable layer on top of the convection zone (Warnecke et al. 2016).

\subsection{A Plume Boundary Condition}

An alternative to an impenetrable boundary condition is a semi-open formulation which permits flows to enter and exit the domain subject to some constraints such as mass conservation, a constant pressure surface, or a specification of some of the flows. These formulations are inherently challenging as they attempt to admit dynamics into the simulation that come from or end up beyond the simulated domain. Semi-open boundaries are successfully used in a variety of codes designed for near-surface solar convection, including MuRAM (Rempel et al. 2009; Cheung et al. 2010), Stagger (Trampedach \& Stein 2011), and CSS (Augustson et al. 2011), though it should be noted that all of these codes are fully compressible and use finite-difference algorithms. ASH uses an anelastic formulation and is pseudo-spectral, which presents additional limitations.

In general, semi-open boundaries tend to generate pressure perturbations which are the result of the flows being specified rather than resulting from solutions of the differential equations being solved. In compressible codes this generates acoustic modes which are often described as box modes, which can often be damped either volumetrically or by use of an absorbing boundary somewhere else in the domain. In ASH, which does not permit acoustic waves, these perturbations can only be dampened by coupling to viscous or thermal diffusion, which restricts the physical and temporal scales over which flows can vary.

In ASH we have chosen to implement what we term a plume boundary condition in which radial velocity and entropy are specified on the top boundary and horizontal velocities and pressure are determined by the interior of the simulation. For full details of 
a)

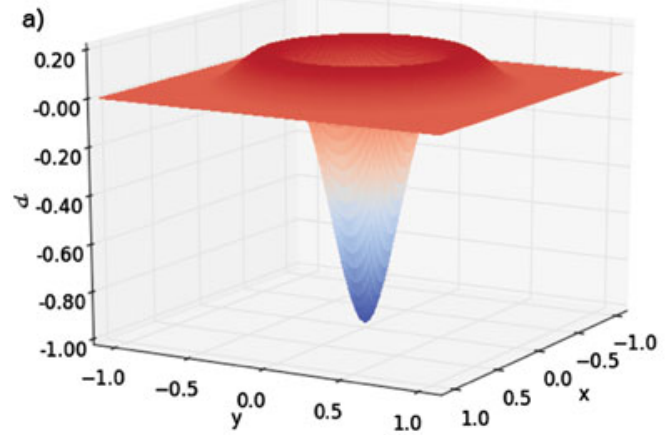

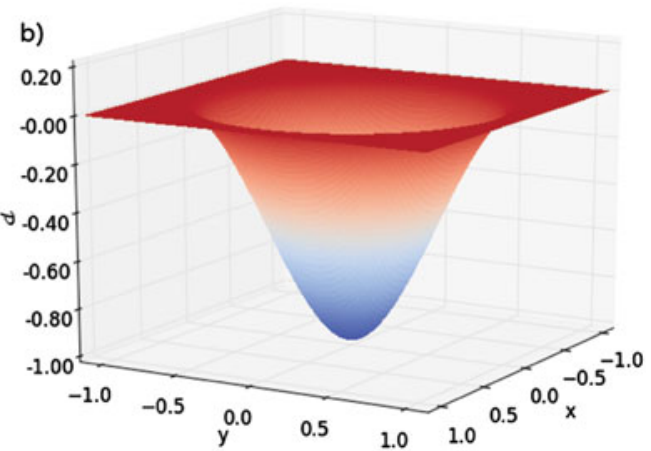

Figure 2. Volume rendering of plume profiles. (a) A so-called "Mexican hat" profile which integrates to zero total contribution. (b) A simpler cold-only plume shape which is offset by a global adjustment to either velocity or thermal fields.

the implementation we refer to Nelson (2013). We choose to populate our boundary with large numbers of small-scale plumes, which are shown in Figure 2. These plumes are local functions with compact support which smoothly match to zero outside of their footprint. The two options explored here are a fifth-order polynomial that gives a "Mexican hat" plume which integrates to zero over a spherical surface, and a third-order polynomial that has a non-zero integral, which we term a cold-only plume. For quantities such as the mass flux or entropy perturbation which we require to be zero the Mexican hat plumes are truly local, while the cold-only plumes require a net outflow or high entropy somewhere else to provide the zero average value we require. We have experimented with a variety of combinations, but the simulation shown here uses Mexican hat shapes for both the radial velocity and entropy profiles of the plumes.

One of the consequences of the plume boundary condition is that it does not locally conserve angular momentum. This allows the global angular momentum of the simulation to vary. To counteract this effect we apply a volumetric torque fitted to counter the time-averaged angular momentum flux through the plume boundary. This assures global angular momentum conservation over long timescales, however it also introduces an effective non-local transport of angular momentum as losses through the boundary are not immediately replaced at the same location. Thus this formulation of the plume boundary condition makes it difficult to study the resulting differential rotation in detail.

Figure 3 shows a snapshot of the plume boundary condition applied to a convective model using four-fold symmetry to save computational expense. The radial velocity and entropy perturbations are specified, while the pressure field is influenced implicitly by these fields as well as the interior of the simulation domain. Generally, however we see that the downflow, low-entropy cores of the plumes correspond to low pressure regions, ensuring that plumes converge as is appropriate in a spherical geometry.

Each individual plume is given its own size, duration, amplitude in both velocity and entropy, and location which are randomly chosen from a specified range. The choices for the simulation presented here are shown in Table 1. The choice of radial velocity and entropy amplitudes are constrained such that a solar luminosity of enthalpy flux is transported through the boundary. Correlations between some of these parameters might reasonably be expected. For example, one might expect larger plumes to be faster, or smaller plumes to have shorter lifetimes. Here we chose to correlate the velocity amplitude, entropy amplitude, plume width, and plume lifetime. Thus each plume is randomly assigned each of the six parameters with the given correlations from the specified range. Finally, we choose to advect longitudinal position of the plume centers $\phi_{p}$ with the mean 

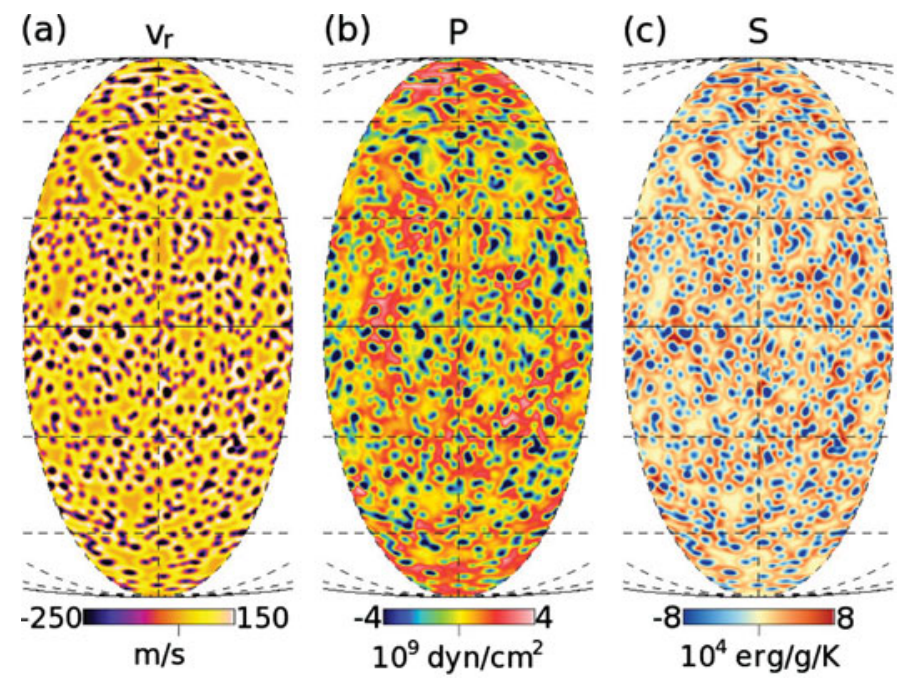

Figure 3. Snapshot of the plume boundary condition applied on the upper boundary of an ASH simulation. The plume boundary condition applies (a) explicit radial velocity structures with a "Mexican-hat" profile, (b) implicit pressure perturbations, and (c) explicit entropy structures, with either a Mexican-hat profile (shown here) or a cold-only profile.

Table 1. Values for Plume Parameters

\begin{tabular}{|c|c|c|c|}
\hline Parameter & Value Range & Units & Correlation \\
\hline $\mathcal{V}$ & {$[240,360]$} & $\mathrm{m} \mathrm{s}^{-1}$ & - \\
\hline $\mathcal{E}$ & {$[1.28,1.56]$} & $10^{5}$ erg $\mathrm{K}^{-1} \mathrm{~g}^{-1}$ & 0.5 \\
\hline$\delta$ & {$[0.08,0.12]$} & rad. & 0.5 \\
\hline$\delta R_{\text {top }}$ & {$[54.6,82.0]$} & $\mathrm{Mm}$ & 0.5 \\
\hline$\tau$ & {$[12.0,18.0]$} & days & 0.5 \\
\hline$\theta_{p}$ & {$[0, \pi]$} & rad. & 0 \\
\hline$\phi_{p}$ & {$[0, \pi / 2]$} & rad. & 0 \\
\hline
\end{tabular}

Notes: Plume boundary parameters used where $\mathcal{V}$ is the peak downflow velocity, $\mathcal{E}$ is the peak entropy perturbation, $\delta$ is the plume's angular radius, $\tau$ is the plume lifetime, and $\theta_{p}$ and $\phi_{p}$ give the coordinate location of the center of the plume on the outer boundary. Also given is the plume width given by $\delta R_{\text {top }}$ in $\mathrm{Mm}$ for ease of comparison. Correlations are expressed with respect to $\mathcal{V}$.

differential rotation. Plumes are initiated with a linear ramp-up phase corresponding to $10 \%$ of their total lifetimes, followed by a constant phase for $80 \%$ of their lifetimes, and a ramp-down phase for the final $10 \%$. After ending their lives they are given new random parameters and restarted at a new location.

\subsection{Coalescence of Plumes into Giant Cells}

The use of the plume boundary condition provides a means to test a long-standing theoretical proposition put forward by Spruit (1997) which argues that giant cell convection is formed not by distributed driving due to a super-adiabatic gradient throughout the convection zone, but instead driving occurs almost exclusively at the photosphere (see also Brandenburg 2016). Convection below the photospheric layers are then a selforganization of plumes. This "entropy rain" model of convection is essentially what is achieved with the plume boundary condition reported here. Because our simulation transports energy through the upper boundary by enthalpy rather than diffusion, it does not create the strong superadiabatic gradients seen in other ASH simulations. In fact compare to a comparable simulation with a closed boundary, this plume boundary simulation's mean entropy gradient is reduced by a factor 10 at the upper boundary, reduced by a 

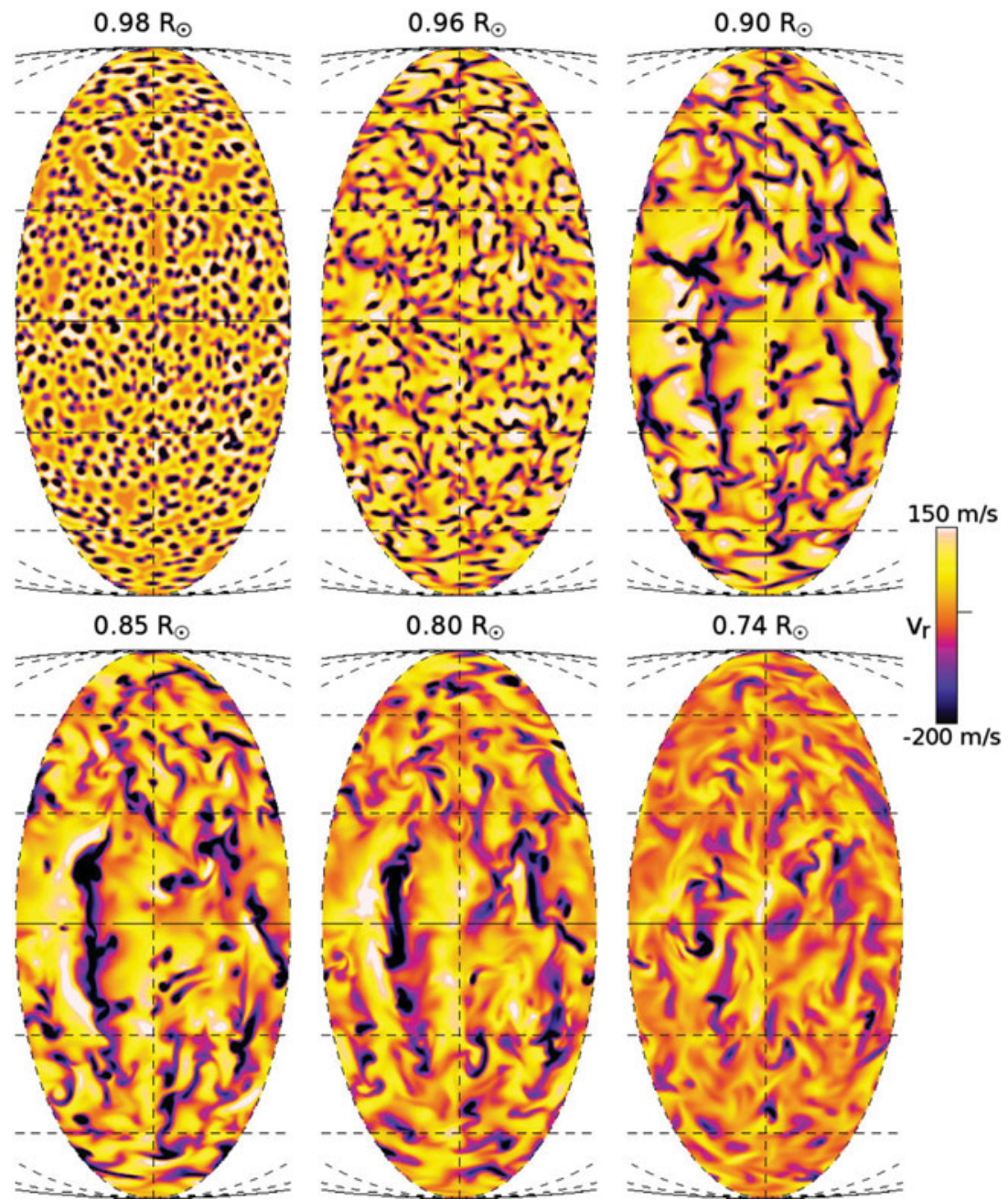

Figure 4. Snapshot of radial velocity at six depths in a simulation using the plume boundary condition with depths indicated. Small-scale, isotropic, randomly-placed plumes are applied on the outer boundary, but quickly coalesce first into sheets with rotational alignment and then into large banana cells with clear rotational alignment.

factor of 2 at mid-convection zone, and is even slightly subadiabatic between $0.93 R_{\odot}$ and $0.97 R_{\odot}$. This essentially removes the majority of the convective driving due to the bulk stratification.

Figure 4 shows a snapshot of the radial velocity at six depths in a well-equilibrated ASH simulation using the plume boundary model with the parameters shown in Table 1. At the boundary plumes are isotropic and very small in scale, but as the plumes descend they rapidly self-organize first into elongated structures resembling sheets at $0.96 R_{\odot}$. By $0.90 R_{\odot}$ these sheets have become rotationally aligned, particularly at low latitudes. Individual downflow plumes can still be seen, but most have merged into much larger scale structures which resemble the "banana cells" seen in closed-boundary models with solar-like differential rotation (e.g., Miesch et al. 2006; Brown et al. 2010; Guerrero \& Käpylä 2011; Gastine \& Wicht 2012; Featherstone \& Miesch 2015). These giant cells are much larger in scale than the plumes and their scale roughly goes at the local pressure 


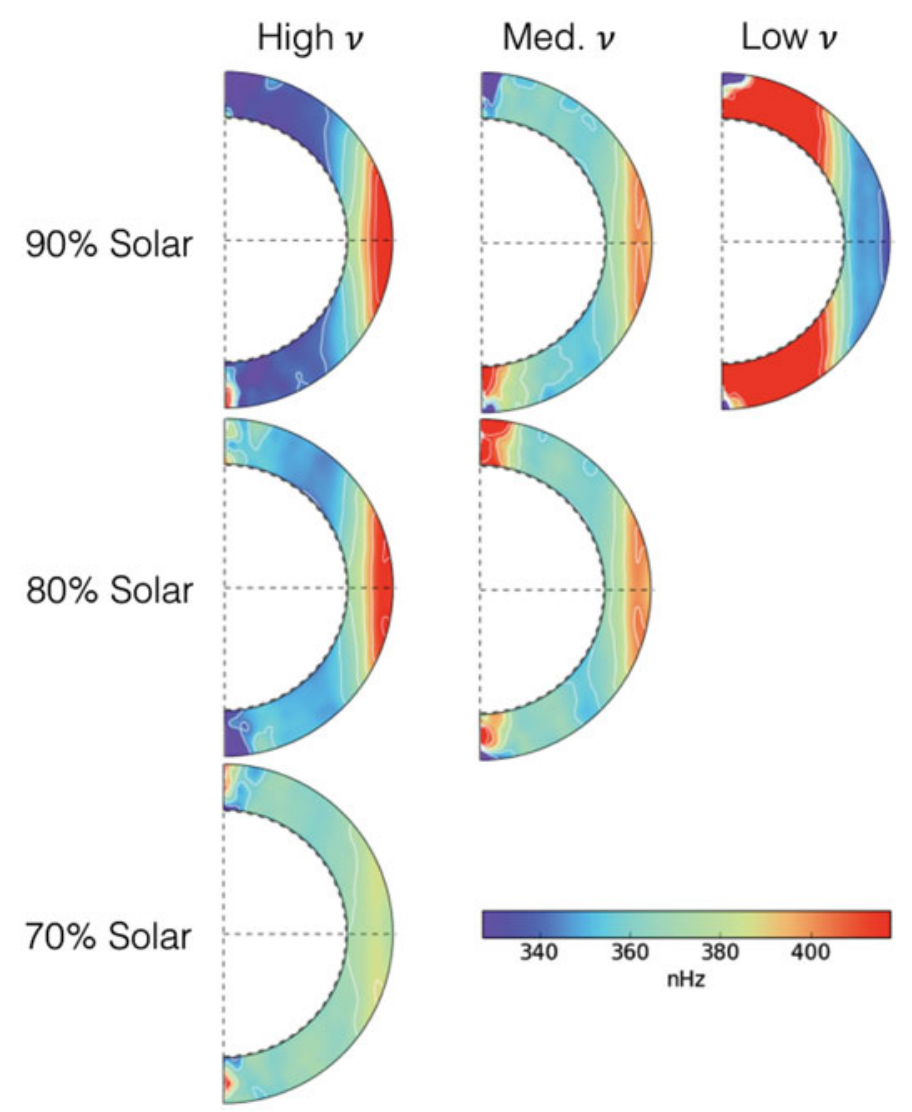

Figure 5. Time-averaged differential rotation profiles for six simulations of solar-like stars varying the bulk rotation rate of the simulation as well as the level of diffusion. Simulations show a systematic trend towards lower differential rotation with decreasing rotation rate, and a transition from solar-like to anti-solar differential rotation for decreasing viscosity $\nu$ at constant Prandtl number.

scale height. Finally near the base of the convection zone the convection slows and is dominated by return flows from the lower boundary.

We appear to have discovered a hybrid between the traditional giant cell model and the entropy-rain model with this plume boundary treatment. In our model we achieve both a strong reduction in the super-adiabatic gradient and yet still achieve convective patterns that morphologically fit the giant cells expected by the traditional model. More study is needed to investigate the properties of these solutions further, but this presents an intriguing possible unification of these two models for convective giant cells in sun-like stars.

\section{The Solar/Anti-Solar Transition in Differential Rotation}

Stellar spin down with age has long been modeled using the Skumanich relationship $\Omega \propto t^{-1 / 2}$ (Skumanich 1972). Recent results using asteroseismic ages have shown that older stars systematically deviate from this trend and show excessively fast rotation rates (van Saders et al. 2016). Specifically, stars that show less rotational constraint on their giant cell convection than the Sun seem to be spinning down more slowly than predicted. Based on these results Metcalfe et al. (2016) have proposed that a transition may occur 
near the solar Rossby number which causes a shift in the dynamo mechanism and thus a change in the rate of angular momentum loss.

Motivated by this observational trend, we have begun to investigate the behavior of stellar differential rotation at rotation rates slower than the current Sun. It has been shown by Gastine et al. (2013) and Featherstone \& Miesch (2015) that lower levels of rotational constraint than our Sun's leads to anti-solar differential rotation profiles with slowly rotating equatorial regions and rapidly rotating mid- to high-latitudes. This anti-solar differential rotation can be understood as a loss of correlation in the Reynold stress transport seen in solar-like differential rotation (see Brun \& Toomre 2002), leading to a mixing of specific angular momentum throughout the convection zone. Previous efforts have focused on changing the Rossby number (the ratio of convective to planetary vorticities) by altering the convective driving by changing the diffusion coefficients of the models or by increasing the rotation rate above the solar value. Here we present some initial results of simulations below the solar rotation rate at a variety of levels of diffusion.

We are conducting an on-going sweep of parameter space of rotation rate and turbulent viscosity with initial results shown here. All simulations are similar to case AB2 of Brun $\&$ Toomre $(2002)$ but extend to $0.98 R_{\odot}$ and do not use different treatments for the spherically-symmetric diffusive transport compared to the diffusion felt by other modes. They are also similar to the 4 scale-height models of Featherstone \& Miesch (2015), but use a solar stratification based on a stellar structure model rather than a polytropic reference state. All models shown here use a closed boundary condition. Here we examine the results of six of these models.

Figure 5 shows initial results form a suite of simulations exploring the relative effects of decreasing diffusion (represented by the viscosity $\nu$ ) and decreasing rotation rate $\Omega$. We see clear trends in differential rotation with both decreasing diffusion and decreasing rotation rate. For our most turbulent case at $0.9 \Omega_{\odot}$ we achieve an anti-solar differential rotation profile. For our slowest rotator at $0.7 \Omega_{\odot}$ we see essentially solid body rotation even over $\sim 200$ years of evolution. This clearly shows that for $3 \mathrm{D}$ global convection models both the bulk rotation rate and the level of convective driving can cause transitions from solar-like to anti-solar differential rotation.

\section{Future Directions}

In this paper we have presented the development and initial use of a plume boundary model for global 3D solar-like convection models. As we continue to gain confidence in the effects of our plume boundary model this treatment may provide some important paths toward better models of stellar dynamo action. To list a few, a plume boundary condition may provide paths toward:

-Improved convective driving in highly turbulent convection simulations, permitting solar-like differential rotation for solar values of the rotation rate and luminosity at levels of turbulence beyond those currently achievable.

-Eventual coupling between global solar convection simulations and local, near-surface models (e.g., Stein \& Nordlund 2012; Leake et al. 2013). Early forms of one-way coupling can take the form of using near-surface models to generate plume statistics for our plume boundary model or applying ASH convective patterns to the bottom of near-surface models. More advanced dynamic coupling schemes may involve tiled near-surface models and global simulations running interactively.

-Buoyant magnetic loops generated by global convective dynamo models (e.g., Nelson et al. 2011; Warnecke et al. 2012; Nelson et al. 2013b) could be simulated much closer to the photosphere than currently permitted. In current closed-boundary models such 
loops are limited to heights below the upper boundary layer. This could be particularly interesting when combined with a coupling to a near-surface simulation, which could then in principle take a flux rope all the way from generation to emergence as an active region.

We have also presented initial results from a suite of models seeking to explore the transition from solar-like to anti-solar differential rotation as a function of both convective driving and bulk rotation rate. Continued exploration at still lower levels of diffusion is needed. Moving forward we will explore the magnetic fields generated by simulations in this parameter space. These varied states of differential rotation should clearly host a wide variety of dynamos. Additionally it has been shown in some cases that the presence of a dynamo can sustain solar-like differential rotation beyond the transition seen in hydrodynamic simulations (Fan \& Fang 2016).

While only one of many issues facing the question of habitability, the ability to predict magnetic activity across a wide range of stellar ages would provide a key step towards a coherent theoretical understanding of life around an active star. In order to have confidence in a dynamo model, it is imperative to resolve questions of the nature of convective driving of giant cells and of the evolution of differential rotation over evolutionary timescales.

\section{Acknowledgements}

The authors would like to thank Juri Toomre, Mark Miesch, and Nicholas Featherstone for many useful discussions related to the ideas presented here. Special additional thanks to Nicholas Featherstone for his development of Rayleigh and allowing us to be early users. Some of this work was conducted while N.J.N. was supported by a Nicholas C. Metropolis Post-Doctoral Fellowship as part of the Advanced Simulation Capabilities Initiative at Los Alamos National Laboratory. C.P. and C.M.S. were supported by the Physics Summer Research Institute at California State University, Chico.

\section{References}

Airapetian, V. S., Glocer, A., Khazanov, G. V., et al. 2017, The Astrophysical Journal, 836, L3 Anglada-Escudé, G., Amado, P. J., Barnes, J., et al. 2016, Nature, 536, 437

Augustson, K., Brun, A. S., Miesch, M., \& Toomre, J. 2015, The Astrophysical Journal, 809, 149

Augustson, K. C., Brun, A. S., \& Toomre, J. 2016, The Astrophysical Journal, 829, 1

Augustson, K. C., Rast, M., Trampedach, R., \& Toomre, J. 2011, Journal of Physics: Conference Series, 271, 012070

Bixel, A., \& Apai, D. 2017, The Astrophysical Journal, 836, L31

Boutle, I. A., Mayne, N. J., Drummond, B., et al. 2017, arXiv preprint, 1

Brandenburg, A. 2016, Astrophysical Journal, 832, 1

Brown, B. P., Browning, M. K., Brun, A. S., Miesch, M. S., \& Toomre, J. 2008, The Astrophysical Journal, 689, 1354

-. 2010, The Astrophysical Journal, 711, 424

Brown, B. P., Miesch, M. S., Browning, M. K., Brun, A. S., \& Toomre, J. 2011, The Astrophysical Journal, 731, 69

Browning, M. K. 2008, The Astrophysical Journal, 676, 1262

Brun, A. S., Miesch, M. S., \& Toomre, J. 2004, The Astrophysical Journal, 614, 1073

Brun, A. S., \& Toomre, J. 2002, The Astrophysical Journal, 570, 865

Brun, A. S., Strugarek, A., Varela, J., et al. 2017, The Astrophysical Journal, 836, 1

Cheung, M. C. M., Rempel, M., Title, A. M., \& Schüssler, M. 2010, The Astrophysical Journal, 720,233 
Clune, T., Elliott, J., Miesch, M. S., Toomre, J., \& Glatzmaier, G. A. 1999, Parallel Computing, 25,361

Coleman, G. A. L., Nelson, R. P., Paardekooper, S. J., et al. 2017, Monthly Notices of the Royal Astronomical Society, arXiv:1608.06908

Fan, Y., \& Fang, F. 2016, Advances in Space Research, 58, 1497

Featherstone, N. A., Browning, M. K., Brun, A. S., \& Toomre, J. 2009, The Astrophysical Journal, 705, 1000

Featherstone, N. A., \& Hindman, B. W. 2016a, The Astrophysical Journal, 830, L15

-. 2016b, The Astrophysical Journal, 818, 32

Featherstone, N. A., \& Miesch, M. S. 2015, The Astrophysical Journal, 804, 1

Garrafo, C., Drake, J. J., \& Cohen, O. 2016, The Astrophysical Journal Letters, 833, 1

Gastine, T., \& Wicht, J. 2012, Icarus, 219, 428

Gastine, T., Yadav, R. K., Morin, J., Reiners, A., \& Wicht, J. 2013, Monthly Notices of the Royal Astronomical Society: Letters, 438, L76

Gillon, M., Jehin, E., Lederer, S. M., et al. 2016, Nature, 533, 221

Gilman, P. 1983, The Astrophysical Journal Supplement Series, 53, 243

Glatzmaier, G. A. 1985, The Astrophysical Journal, 291, 300

Greer, B. J., Hindman, B. W., Featherstone, N. a., \& Toomre, J. 2015, The Astrophysical Journal, 803, L17

Greer, B. J., Hindman, B. W., \& Toomre, J. 2014, Solar Physics

Guerrero, G., \& Käpylä, P. J. 2011, Astronomy \& Astrophysics, 533, A40

Hanasoge, S. M., Duvall, T. L., \& DeRosa, M. L. 2010, The Astrophysical Journal, 712, L98

Hanasoge, S. M., Duvall, T. L., \& Sreenivasan, K. R. 2012, Proceedings of the National Academy of Sciences, 109, 11928

Hathaway, D. H., Upton, L., \& Colegrove, O. 2013, Science (New York, N.Y.), 342, 1217

Kane, S. R., Gelino, D. M., \& Turnbull, M. C. 2017, The Astronomical Journal, 153, 52

Leake, J. E., Linton, M. G., \& Torok, T. 2013, The Astrophysical Journal, 778, 12

Luger, R., Lustig-Yaeger, J., Fleming, D. P., et al. 2016, eprint arXiv:1609.09075, 837, 1

McIntosh, S. W., Wang, X., Leamon, R. J., \& Scherrer, P. H. 2014, The Astrophysical Journal, 784, L32

Méndez, A., \& Rivera-Valentín, E. G. 2017, The Astrophysical Journal Letters, 837, 1

Metcalfe, T. S., Egeland, R., \& van Saders, J. L. 2016, The Astrophysical Journal, 826, L2

Miesch, M. S., Brun, A. S., \& Toomre, J. 2006, The Astrophysical Journal, 641, 618

Nelson, N. J. 2013, Proquest dissertations \& theses: 3607343, University of Colorado

Nelson, N. J., Brown, B. P., Brun, A. S., Miesch, M. S., \& Toomre, J. 2011, The Astrophysical Journal, 739, L38

-. 2013a, The Astrophysical Journal, 762, 73

Nelson, N. J., Brown, B. P., Sacha Brun, a., Miesch, M. S., \& Toomre, J. 2013b, Solar Physics

Nelson, N. J., \& Miesch, M. S. 2014, Plasma Physics and Controlled Fusion, 56, 064004

Reiners, A. 2012, Living Reviews in Solar Physics, 8, 1

Rempel, M., Schüssler, M., \& Knölker, M. 2009, The Astrophysical Journal, 691, 640

Skumanich, A. 1972, The Astrophysical Journal, 171, 565

Spruit, H. 1997, Mem. Soci. Astron. Ital., 68, 397

Stein, R. F., \& Nordlund, A. k. 2012, The Astrophysical Journal, 753, L13

Trampedach, R., \& Stein, R. F. 2011, The Astrophysical Journal, 731, 78

van Saders, J. L., Ceillier, T., Metcalfe, T. S., et al. 2016, Nature, 529, 181

Warnecke, J., Käpylä, P. J., Käpylä, M. J., \& Brandenburg, A. 2016, Astronomy \& Astrophysics, 596, A115

Warnecke, J., Käpylä, P. J., Mantere, M. J., \& Brandenburg, A. 2012, Solar Physics, 280, 299

Yadav, R. K., Christensen, U. R., Wolk, S. J., \& Poppenhaeger, K. 2016, The Astrophysical Journal, 833, L28 